Received: $\quad 2019.10 .18$

Accepted: 2019.12.27 Available online: 2020.01 .30 Published: 2020.03 .23

\title{
Long Noncoding RNA (IncRNA) Maternally Expressed Gene 3 (MEG3) Participates in Chronic Obstructive Pulmonary Disease through Regulating Human Pulmonary Microvascular Endothelial Cell Apoptosis
}

Authors' Contribution: Study Design A Data Collection B Statistical Analysis C Data Interpretation D Manuscript Preparation E Literature Search F Funds Collection G
ABCDEFG 1 Hui Bi

BCDF 2 Gui Wang

BCD 1 Zhiying Li

$B C D 1$ Lin Zhou

BCD 1 Ming Zhang

BF 1 Jiru Ye

BF 1 Zhigang Wang
1 Department of Respiratory Medicine, The Third Affiliated Hospital of Soochow University, Changzhou, Jiangsu, P.R. China

2 Department of Intensive Care Unit, The Third Affiliated Hospital of Soochow University, Changzhou, Jiangsu, P.R. China
Corresponding Author:

Source of support:
Hui Bi, e-mail: bihui101619@163.com

The present study was supported by the Youth Talents Science and Technology Project of Changzhou Health Commission (No. QN201904)

Background: Chronic obstructive pulmonary disease (COPD), a general airway disease, is featured by progressive and chronic immunoreaction in the lung. Increasing evidences have showed that cigarette smoking is the main reason in the COPD progression, and human pulmonary microvascular endothelial cell (HPMEC) apoptosis often be observed in COPD, while its pathogenesis is not yet fully described. Upregulation of long noncoding RNA (IncRNA) maternally expressed gene 3 (MEG3) was observed in COPD patients, but the specific mechanism of IncRNA MEG3 in COPD remains unknown. The objective of this research was to explore the role of IncRNA MEG3 in cigarette smoke extract (CSE)-induced HPMECs.

Material/Methods: $\quad$ HPMECs were induced by a series of concentrations of CSE $(0 \%, 0.1 \%, 1 \%$, and $10 \%)$. Then cell apoptosis was analyzed by flow cytometry. Cell apoptosis related proteins were tested using western blot assay. Finally, we applied knockdown and over-expression system to explore the IncRNA MEG3 functions in CSE-induced HPMECs.

Results: Our results indicated that various concentrations of CSE $(0 \%, 0.1 \%, 1 \%$, and $10 \%)$ significantly promoted cell apoptosis, augmented caspase- 3 activity, upregulated Bax expression, decreased Bcl-2 expression, and enhanced IncRNA MEG3 level in HPMECs. LncRNA MEG3-plasmid transfection resulted in the upregulation of IncRNA MEG3, more apoptotic HPMECs, and higher caspase-3 activity. While IncRNA MEG3 knockdown presented the opposite effects. Further investigation suggested that all the effects of CSE treatment on HPMECS were markedly reversed by IncRNA MEG3-shRNA (short hairpin RNA).

Conclusions: $\quad$ Our study illustrated a protective effect of IncRNA MEG3-shRNA on CSE-induced HPMECs, indicting IncRNA MEG3 can be a new therapeutic approach for COPD treatment.

MeSH Keywords: $\quad$ Apoptosis • Pulmonary Disease, Chronic Obstructive • RNA, Long Noncoding

Full-text PDF: https://www.medscimonit.com/abstract/index/idArt/920793

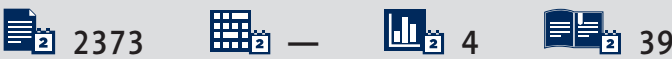




\section{Background}

Chronic obstructive pulmonary disease (COPD) is a chronic respiratory disease featured by irreversible debility of lung function [1]. As a main global health disease, COPD has been predicted to become the fourth primary causing death worldwide by 2020 [2]. Plenty of evidence have demonstrated that many elements were participated in the progression of COPD, such as genetic susceptibility [3], environmental risk [4] and abnormal inflammatory response [5]. Cigarette smoking, one of the predominant risk factors of COPD, may result in increasing cell apoptosis, damaging endothelial cells and more inflammatory response in lung [6]. Many evidences have indicated that more apoptotic cells were exist in COPD lungs than normal control, while its nosogenesis remains unexplored [7]. He et al. found that cigarette smoke extract promoted human bronchial epithelial cells apoptosis [8]. However, few effective therapeutic targets of COPD were found, and it is crucial to explore the detailed mechanism of COPD.

Previous studies have been reported that many genes participated in the COPD progression, containing microRNAs (miRNAs) and long noncoding RNAs (IncRNAs) [9]. LncRNA, a cluster of RNA transcripts with more than 200 nucleotides in length, were participated in mediating multiple cell physiological progress, including cell proliferation, apoptosis and migration [10]. In recent years, Li et al. found that InCRNA HEIH affected triple-negative breast cancer cells growth and apoptosis through regulating miR-4458/SOCS1 axis [11]. Wu et al. demonstrated that IncRNA ZEB2-AS1 regulates bladder cancer cell growth and apoptosis through miR-27b [12]. The IncRNA maternally expressed gene 3 (MEG3), one kind of IncRNA, is found to be a tumor inhibitor and existed in many normal organs [13]. Previous reports have illustrated that IncRNA MEG3 was over-expressed in many diseases, including, cancer retinopathy, osteoarthritis and cardiovascular disease [14-17]. LncRNA MEG3 can regulate many types of cellular functions, including cell viability and apoptosis $[16,18,19]$. However, the interaction mechanism between InCRNA MEG3 and COPD need to be fully illuminated. Studies have revealed that human pulmonary microvascular endothelial cell (HPMEC) apoptosis is essential in the occurrence and development of COPD [20,21]. Thus, we speculated that InCRNA MEG3 may play a key role in COPD through regulating HPMEC apoptosis.

In present report, we explored the function of IncRNA MEG3 in cigarette smoking extract (CSE)-stimulated HPMECs. We evaluated the effects of CSE on cell apoptosis, caspase-3 activity, apoptosis-related protein expression and IncRNA MEG3 levels in HPMECs. Our findings also demonstrated the mechanism of IncRNA MEG3-plasmid or IncRNA MEG3-shRNA (short hairpin RNA) in CSE-stimulated the apoptosis of HPMECs. In addition, we further elaborated the effects of IncRNA MEG3-shRNA on the influence of CSE in HPMECs. To conclude, our results demonstrated the protective function of InCRNA MEG3-shRNA in CSE-treated HPMECs, which may be an underlying target for COPD treatment in clinical.

\section{Material and Methods}

\section{Cell culture}

HPMECs, purchased from ATCC (Manassas, VA, USA), were cultivated in endothelial cell medium (Gibco, USA) containing 10\% heated-inactivated fetal bovine serum (FBS, Gibco), $1 \%$ endothelial cell growth factor (Beyotime, China), $100 \mathrm{IU} / \mathrm{mL}$ penicillin and streptomycin (Sigma) at $37^{\circ} \mathrm{C}$ in a humidified atmosphere of $5 \% \mathrm{CO}_{2}$.

\section{Cigarette smoke extract (CSE) preparation and treatment}

CSE was obtained using a modified method, as previously reported [22]. In brief, one commercial cigarette was burned with a reformative syringe-driven equipment. Then the smoke was bubbled in $20 \mathrm{~mL}$ medium at a speed of $100 \mathrm{~mL} /$ minute for more than 5 minutes. Thereafter, $0.2 \mu \mathrm{m}$ pore membrane filter was conducted to eliminate big particulates from the resulting collection. This isolate was defined as " $100 \%$ CSE".

The $100 \%$ CSE was diluted with phosphate-buffered saline (PBS), then HPMECs were treated with various concentration of CSE $(0.1 \%, 1 \%, 10 \%)$ for 24 hours respectively [23]. Cells without any CSE treatment were regarded as the control group (Control).

\section{Cell transfection}

Synthetic control-plasmid (empty pcDNA3.1 vector; Shanghai GeneChem Co., Ltd.), IncRNA MEG3-plasmid (conducted refer to MEG3 sequence and then sub-cloned into the pcDNA3.1 vector; Shanghai GeneChem Co., Ltd.), control-shRNA (the scrambled shRNA plasmid; PGFP-C-shLenti Vector; cat. no. TR30021) and IncRNA MEG3-shRNA (sh-MEG3 sequence, 5'-GAGAGGTTGTTTCACTGGTATCTATTGCA-3'; pGFP-C-shLenti Vector; cat. no. TL 320132C) were obtained from OriGene. For transfection, HPMECs were incubated into 6-well plates and then transfected with control-plasmid, InCRNA MEG3plasmid, control-shRNA or IncRNA MEG3-shRNA respectively by Lipofectamine 2000 agentia (Beyotime) for 24 hours referring to the manufacturer's protocols.

\section{Flow cytometry analysis}

HPMECs were cultured in 6-well plates. After treatment, cells were obtained using EDTA-trypsin and washed with PBS. 
Then the Annexin V-FITC/propidium iodide (AV/PI) dual staining (Sigma, USA) was performed to determine the HPMECs cell apoptosis following the manufacturer's manual. The apoptotic cells were measured by FACS flow cytometer (BD Biosciences) and analyzed by the Flowjo software (Becton Dickinson, CA, USA).

\section{Detection of caspase-3 activity}

Caspase-3 Colorimetric Assay Kit (Beyotime, Shanghai, China) were used to assess the caspase- 3 activity in HPMECs referring to the manufacturer's protocols. In a word, cells were dissolved with lysis buffer, and the supernatant of the lysate was gathered and centrifuged. Then the supernatant was cultured in Ac-DEVE-pNA and reaction buffer at $37^{\circ} \mathrm{C}$ for 2 hours. A microplate reader (BioTek, VT) was performed to detect optical density (OD) of caspase-3 activity at $405 \mathrm{~nm}$.

\section{Quantitative reverse transcription PCR (qRT-PCR) analysis}

After indicated treatment, total RNA from HPMECs was obtained using RNA-isolation kit (Vazyme) referring to the manufacturer's protocol. RNA was reversely subjected to cDNA using the PrimeScipt RT reagent Kit (Takara, Shiga, Japan). The expression of relative genes was assessed by ABI 7500 Real-time PCR Detection System (Applied Biosystems, USA) with the SYBR Premix Ex Taq (Takara). Human 18srRNA was used as the internal inference for IncRNA. Primers were compounded by Sangon Biotech (Shanghai, China). Relative gene expression was analyzed using the $2^{-\Delta \Delta c t}$ method.

\section{Western blot analysis}

After indicated treatment, total proteins in HPMECs were obtained and extracted using radioimmunoprecipitation assay (RIPA) buffer (Sigma, USA) following the manufacturer's protocol. The lysates concentrations were evaluated by the bicinchoninic acid (BCA) Protein Assay Kit (Thermo). Then the protein extract was mingled with $5 x$ loading buffer and separated by $10 \%$ sodium dodecyl sulfate-polyacrylamide gel electrophoresis (SDS-PAGE) followed by transferring to polyvinylidene difluoride (PVDF) membrane. After that, 5\% fat-free milk was used to block membrane for 1.5 hour at room temperature, and cultivated overnight at $4^{\circ} \mathrm{C}$ with primary antibodies against Bax (Abcam, 1: 1000), Bcl-2 (Abcam, 1: 1000) and $\beta$-actin (1: 1500, Santa Cruz Biotechnology, Inc). The membrane was then washed with PBS with Tween (PBST) and incubated with secondary antibodies (1: 2000, Santa Cruz, USA) for 1 hour at $37^{\circ} \mathrm{C}$. Finally, immunoreactive proteins were visualized by electrochemiluminescence (ECL) detection system (Bio Rad) according to the manufacturer's protocol and quantified with ImageJ Software.

\section{Statistical analysis}

GraphPad Prism 6.0 was conducted to evaluate the statistical analysis. All results were represented as means \pm standard deviation (SD) from 3 individual tests. Statistical comparisons were evaluated using unpaired Student $t$-test or one-way analysis of variance (ANOVA). ${ }^{*} P<0.05$ and ${ }^{* *} P<0.01$ were considered to indicate statistically significant difference.

\section{Results}

\section{CSE stimulated cell apoptosis and enhanced IncRNA MEG3 expression in HPMECs}

HPMECs were stimulated with $0.1 \%, 1 \%$, and $10 \%$ CSE for 24 hours, then we assessed the effects of CSE on HPMECs. First, flow cytometry assay was employed to detect HPMEC apoptosis. Our results suggested that the cell apoptosis increased remarkably after CSE $(0.1 \%, 1 \%$, and $10 \%)$ treatment compared to the control group (Figure 1A). Moreover, the percentage of apoptotic HPMECs was increased in CSE-treated group compared to the control group (Figure 1B). Besides, we found the caspase- 3 activity was significantly promoted after $0.1 \%$ CSE, $1 \%$ CSE, and $10 \%$ CSE treatment for 24 hours compared to that stimulated by $0 \%$ CSE (Figure 1C). Cell apoptosis often mediated by apoptosis-specific proteins, such as Bcl-2 and Bax. Thereafter, Bcl-2 and Bax levels in different groups was evaluated using western blot. As illustrated in Figure 1D, CSE exposure obviously enhanced the Bax expression level and induced a reduce in $\mathrm{Bcl}-2$ in HPMECs. In order to expound the functions of IncRNA MEG3 in CSE-treated HPMECs, we first determined IncRNA MEG3 level by qRT-PCR analysis in CSEstimulated HPMECs. The results revealed that InCRNA MEG3 were apparently upregulated in HPMECs stimulated by $0.1 \%$ to $10 \%$ CSE (Figure 1E). No obvious difference was observed between $1 \%$ and $10 \%$ CSE-induced groups in aforementioned results; thus, we chose $1 \%$ CSE to induce cells for 24 hours in further experiments.

\section{Over-expression of IncRNA MEG3 promoted HPMEC apoptosis}

Previous results revealed that IncRNA MEG3 was upregulated in HPMECs. To lucubrate the potential mechanism of IncRNA MEG3 in cell apoptosis, control-plasmid or IncRNA MEG3plasmid was transfected into HPMECs for 24 hours. Transfection efficiency was evaluated using qRT-PCR. qRT-PCR analytical data revealed that InCRNA MEG3 was upregulated in IncRNA MEG3 plasmid treated HPMECs (Figure 2A). The flow cytometry assay showed that IncRNA MEG3 over-expression increased apoptotic HPMECs remarkably and elevated the percentage of apoptotic cells compared with control group (Figure 2B, 2C). 
A
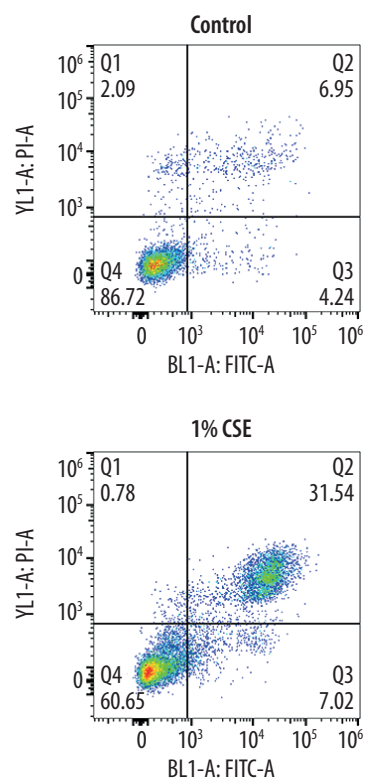

C

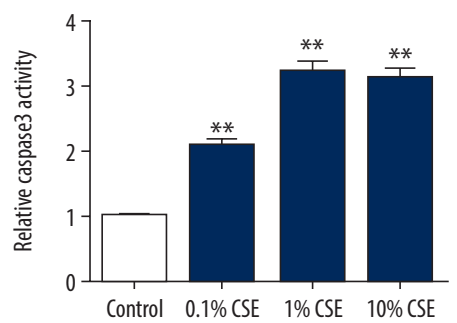

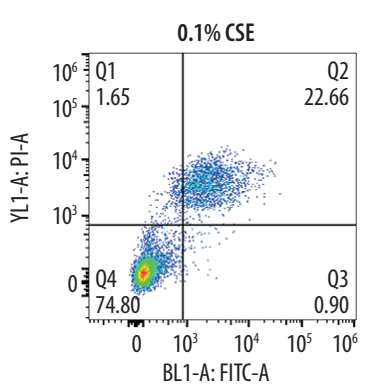

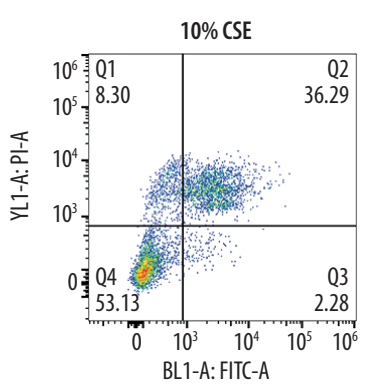

D

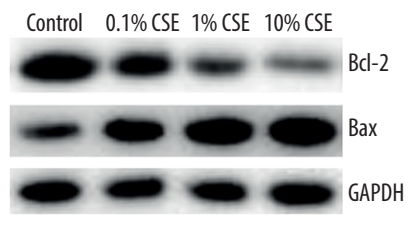

B

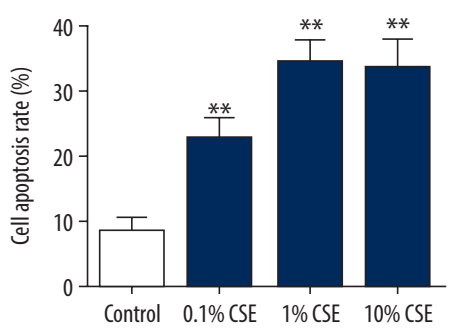

E

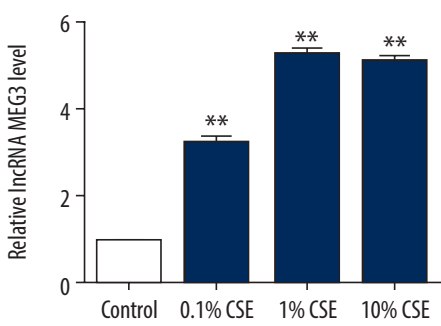

Figure 1. CSE promoted cell apoptosis and increased IncRNA MEG3 expression in HPMECs. HPMECs were subjected to varied concentrations of CSE ( $0 \%, 0.1 \%, 1 \%$, and $10 \%$ CSE) for 24 hours. (A) Flow cytometry assay was adopted to determine the influence of CSE on HPMECs apoptosis. (B) Percentages of apoptotic cells are quantified. (C) Caspase-3 expression was assessed in CSE-treated HPMECs by Caspase-3 Activity Assay Kit. (D) Bax and Bcl-2 levels in CSE-stimulated HPMECS were assessed using western blot. (E) The level of InCRNA MEG3 in CSE-induced HPMECs was measured by qRT-PCR.

${ }^{*} P<0.05$; ${ }^{* *} P<0.01$. CSE - cigarette smoking extract; IncRNA - long noncoding RNA, MEG3 - maternally expressed gene 3; HPMEC - human pulmonary microvascular endothelial cell; qRT-PCR - quantitative reverse transcription PCR.

Furthermore, the caspase-3 activity assay showed that the caspase- 3 activity was significantly increased in IncRNA MEG3 over-expressed HPMECs (Figure 2D). In summary, these data clarified that over-expression of IncRNA MEG3 could induce apoptosis in HPMECs.

\section{Downregulation of IncRNA MEG3 inhibited apoptosis in HPMECS}

For further exploration, we investigated the specific role of IncRNA MEG3 in HPMECs. Control-shRNA or IncRNA MEG3shRNA were transfected into HPMECs. After transfection, IncRNA MEG3 levels in HPMECs was determined by qRT-PCR. We found that IncRNA MEG3 level was dramatically decreased in HPMECs after IncRNA MEG3-shRNA transfection compared to control (Figure $3 \mathrm{~A}$ ). In addition, flow cytometry results revealed that IncRNA MEG3-shRNA-treated HPMECs revealed a significantly decrease in apoptosis and apoptotic cell rate (Figure 3B, 3C). Likewise, compared to the control group, knockdown of MEG3 obviously suppressed the caspase-3 activity in IncRNA MEG3-shRNA-treated HPMECs (Figure 3D). These data clarified that IncRNA MEG3 might be participated in mediating HPMEC apoptosis.

\section{LnCRNA MEG3-shRNA relieved the influence of CSE on HPMECS}

To deeply explore the role of IncRNA MEG3-shRNA in the CSEinduced HPMECs, control-shRNA or IncRNA MEG3-shRNA were transfected into HPMECs for 24 hours, followed by $1 \%$ CSE 


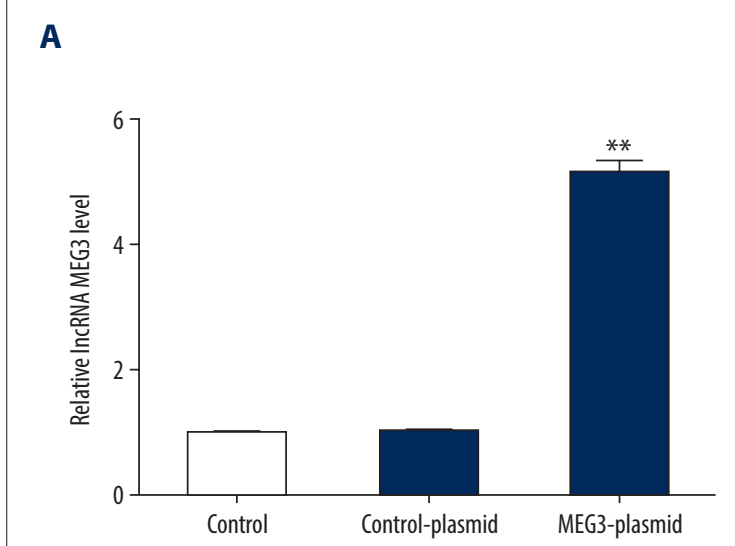

B
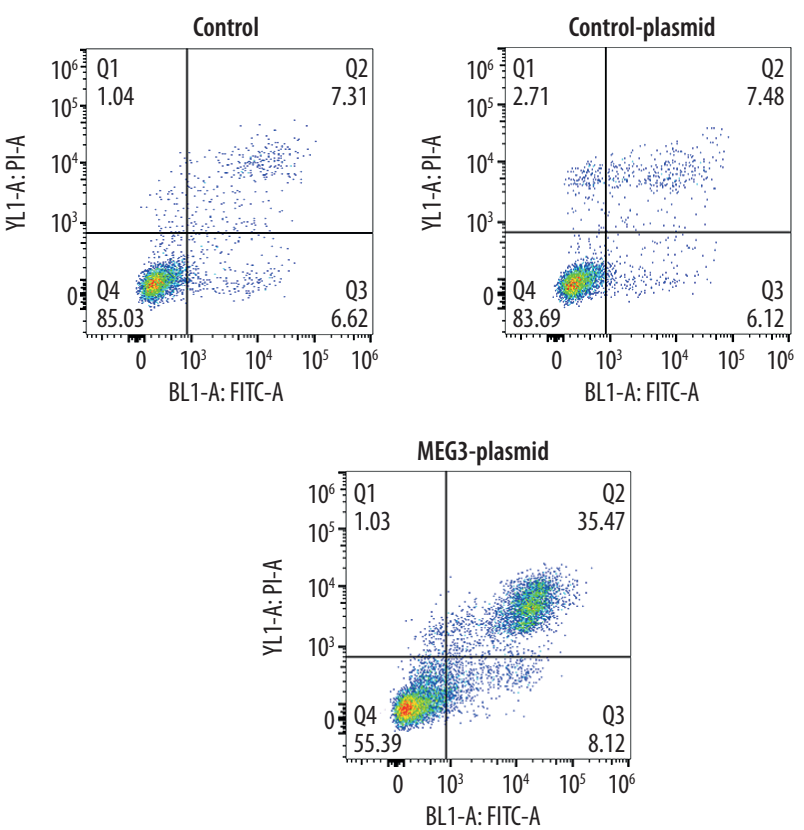

C

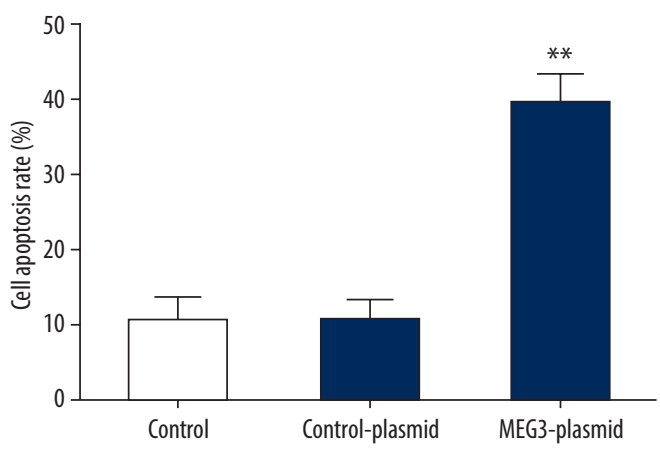

D

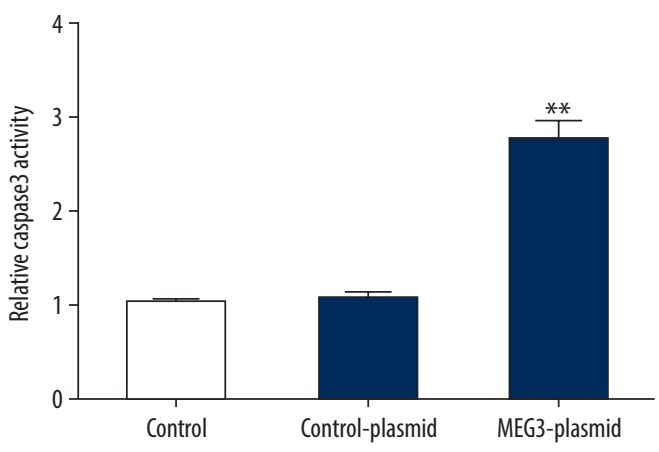

Figure 2. Upregulation of IncRNA MEG3 promoted cell apoptosis in HPMECs. Control-plasmid or IncRNA MEG3-plasmid were transfected into HPMECs for 24 hours and the transfection efficiency was evaluated by qRT-PCR. (A) qRT-PCR analysis of the level of IncRNA MEG3 in HPMECs after transfection. (B) Effect of IncRNA MEG3-plasmid on HPMECs apoptosis was detected by the flow cytometry assay. (C) Percentages of apoptotic cells were quantified. (D) Influence of IncRNA MEG3-plasmid on HPMEC caspase- 3 activity was determined by the caspase- 3 assay kit. ${ }^{*} P<0.05$; ${ }^{\star *} P<0.01$. IncRNA - long noncoding RNA; MEG3 - maternally expressed gene 3; HPMEC - human pulmonary microvascular endothelial cell; qRT-PCR - quantitative reverse transcription PCR.

treatment for another 24 hours. As shown in Figure 4A, the level of IncRNA MEG3 significantly enhanced in CSE-stimulated HPMECs compared to the control group. However, compared with CSE+control-shRNA group, the level of IncRNA MEG3 was significantly depressed in CSE+IncRNA MEG3-shRNA group. Besides, HPMEC apoptosis was detected and the apoptosis rate was also quantified in different groups. More apoptotic cells were found in CSE-treated HPMECs, and the apoptotic rate of HPMECs was higher in the $1 \%$ CSE treatment group compared to the control group. while IncRNA MEG3-shRNA significantly inhibited HPMEC apoptosis (Figure 4B, 4C). Furthermore, the effect of $1 \%$ CSE on caspase-3 activity and apoptosis-related proteins levels were also detected in different groups. Our results revealed that $1 \%$ CSE significantly promoted the caspase-3 activity (Figure 4D), decreased Bcl-2 and increased the Bax expression levels (Figure 4E). Conversely, IncRNA MEG3shRNA reversed all these effects of $1 \%$ CSE on caspase- 3 activity and apoptosis-related genes expression. In summary, these results indicated that InCRNA MEG3-shRNA could reverse the cell apoptosis in CSE-induced HPMECs, indicting IncRNA MEG3 might be a novel target for COPD treatment. 

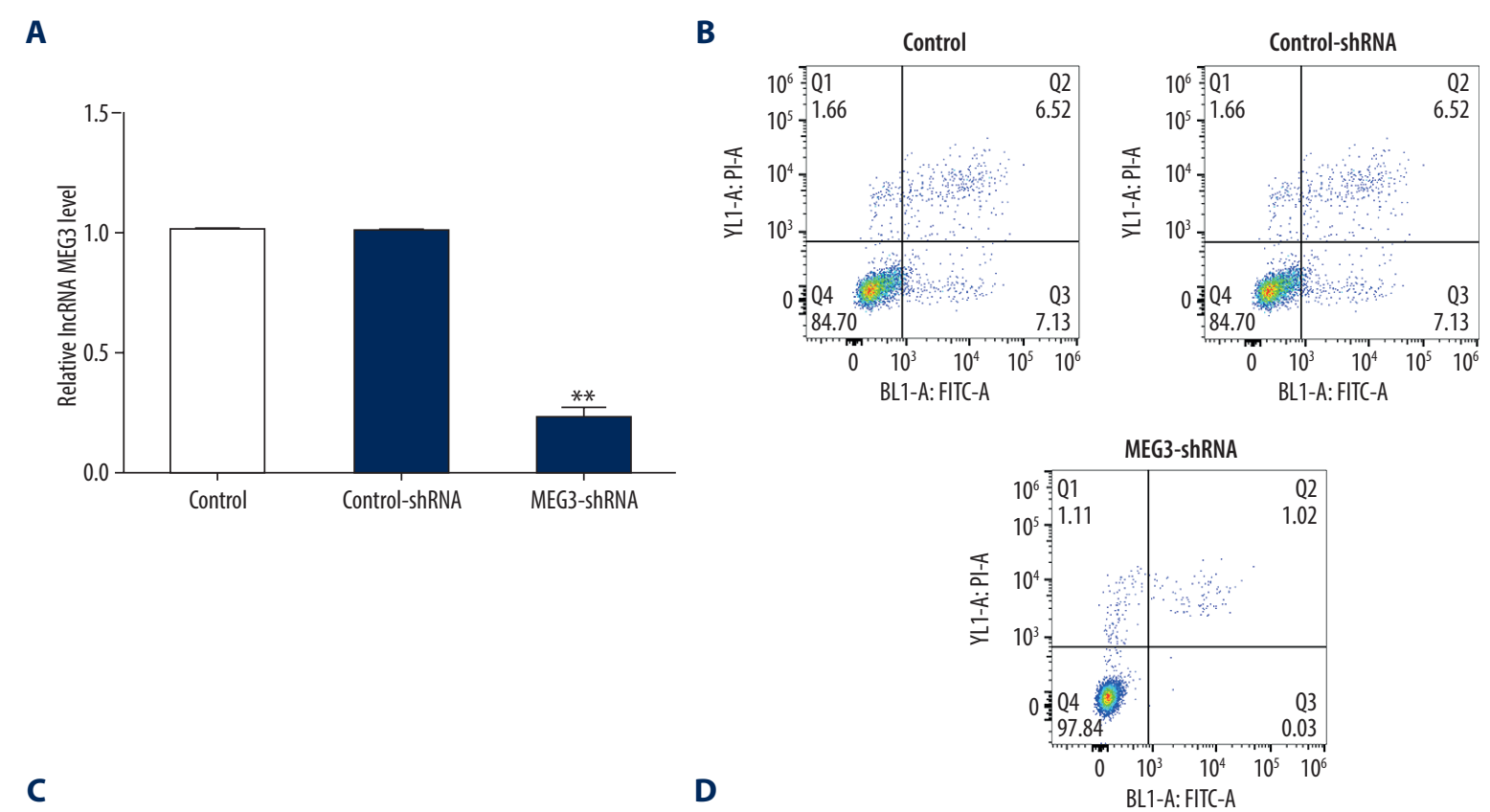

C

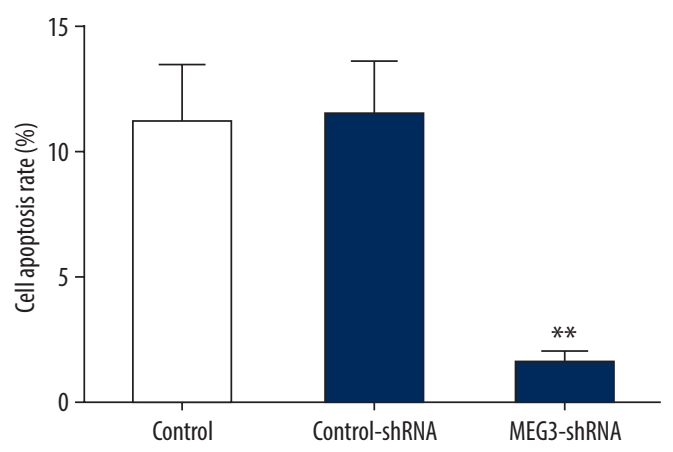

D

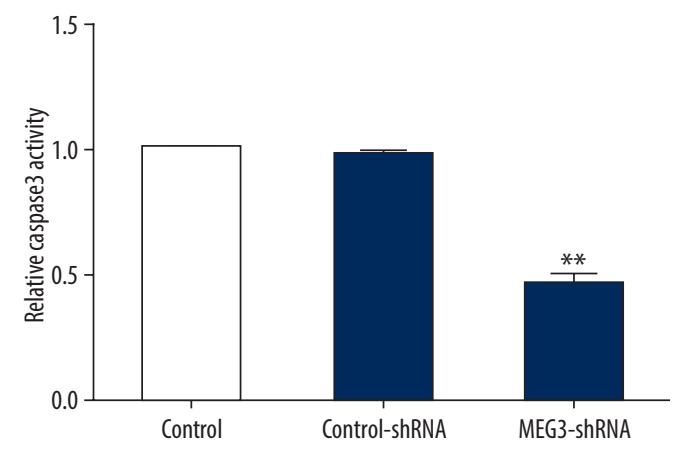

Figure 3. Suppression of InCRNA MEG3 decreased cell apoptosis in HPMECs. HPMECs were transfected with control-shRNA or IncRNA MEG3-shRNA for 24 hours and the transfection efficiency was measured by qRT-PCR. (A) qRT-PCR was conducted to determine the level of IncRNA MEG3 in HPMECs after transfection. (B) Flow cytometry analysis showed the effect of IncRNA MEG3-shRNA on HPMEC apoptosis. (C) Percentages of apoptotic cells are calculated. (D) Effect of IncRNA MEG3-shRNA on HPMEC caspase- 3 activity was assessed by the caspase- 3 assay kit. ${ }^{*} P<0.05$; ${ }^{* *} P<0.01$. IncRNA - long noncoding RNA; MEG3 - maternally expressed gene 3; HPMEC - human pulmonary microvascular endothelial cell; shRNA - short hairpin RNA.

\section{Discussion}

COPD, the fourth primary factor of death around the world, is a chronic disease with poor survival prognosis [24-26]. Evidence showed that cigarette smoking is main hazards in COPD progression, and it could regulate various cells apoptosis including lung epithelial cells and vascular endothelial cells $[27,28]$. Besides, previous reports delineated that increased apoptosis was observed both in COPD patients and animal models $[29,30]$. Thus, in our experiments, we sought to determine the role of CSE in HPMEC apoptosis. Our results suggested that different CSE $(0.1 \%, 1 \%, 10 \%)$ obviously promoted HPMECs apoptosis. As we all know, cell apoptosis is associated with several cascade proteins and apoptosis-related proteins [31]. Consistent with this concept, we detected the caspase- 3 activity and the members of Bax and $\mathrm{Bcl}-2$ family protein levels. We found that CSE increased caspase-3 activity, enhanced Bax levels and reduced $\mathrm{BCl}-2$ expression in HPMECs, especially at the concentration of $1 \%$.

Numerous scientific evidences have indicated that IncRNAs are upregulation in multiple diseases, including Alzheimer's disease [32], coronary artery disease [33] and lung cancer [34], but the relevance between IncRNA and COPD need to be further illustrated. LncRNA MEG3, a new element of IncRNA, is identified as a regulator in cell biological behavior [35] and widely 


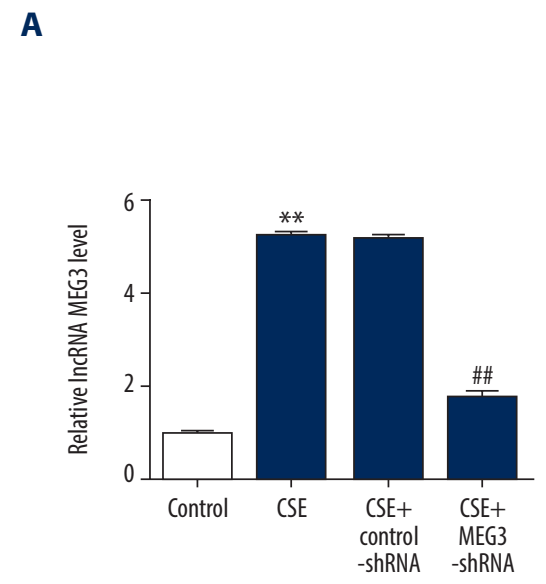

B
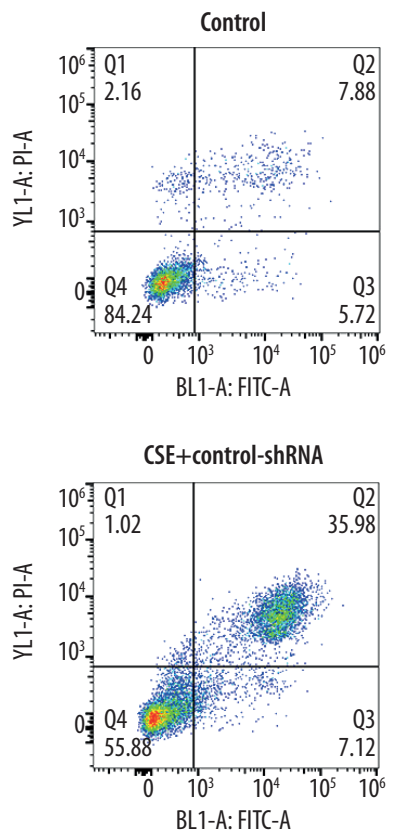

C

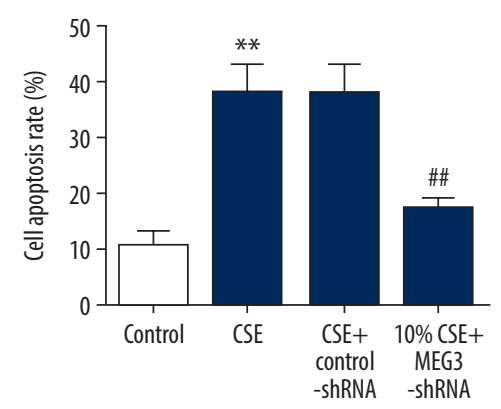

D

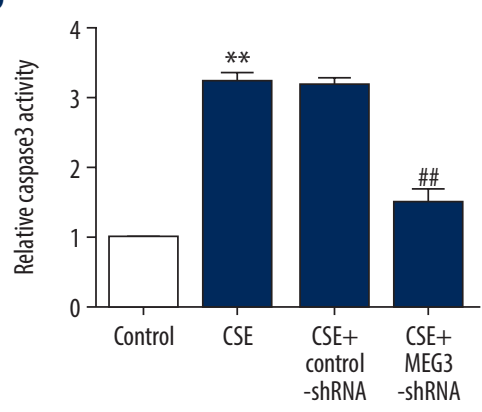

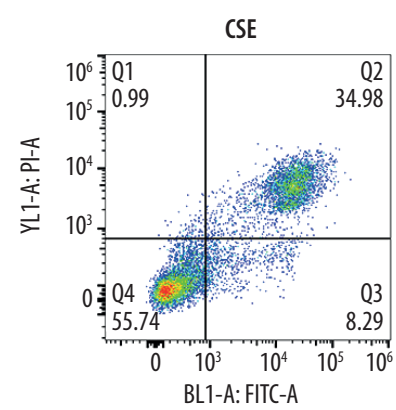

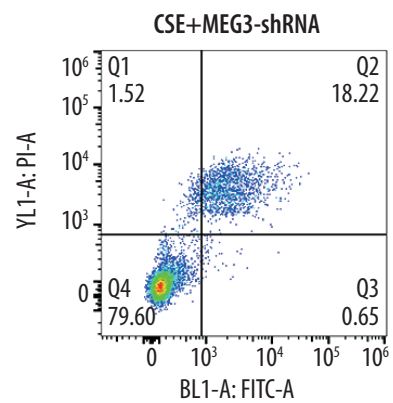

$\mathbf{E}$

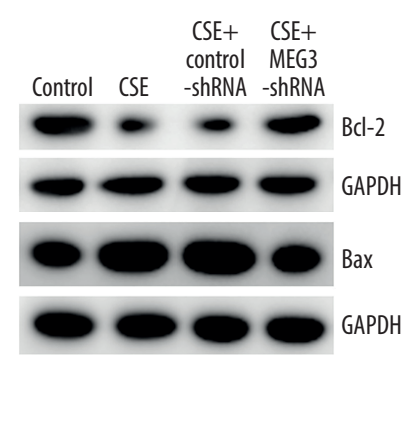

Figure 4. Silencing InCRNA MEG3 reversed the effects of CSE on HPMECs. HPMECs were transfected with control-shRNA or IncRNA MEG3-shRNA for 24 hours, followed by $1 \%$ CSE treatment for another 24 hours. Cells were divided into 4 groups: Control, CSE, CSE+contro-shRNA, and CSE+IncRNA MEG3-shRNA. (A) qRT-PCR analysis showed the level of IncRNA MEG3 in HPMECs in different groups. (B) Cell apoptosis of HPMECs was examined by the flow cytometry assay in different groups. (C) Statistical analysis was represented. (D) Caspase-3 activity of HPMECs was evaluated by the caspase-3 assay kit under different conditions. (E) Western blot assay was applied for measurement of protein expression levels in HPMECs in different groups including $\mathrm{Bax}$ and $\mathrm{BCl}-2$. ${ }^{*} P<0.05 ;{ }^{* *} P<0.01$. IncRNA - long noncoding RNA; MEG3 - maternally expressed gene 3; CSE - cigarette smoking extract; HPMEC - human pulmonary microvascular endothelial cell; shRNA - short hairpin RNA; qRT-PCR - quantitative reverse transcription PCR.

involved in many diseases [14-17]. For example, Zhang et al. found IncRNA MEG3 suppressed cell EMT through sponging miR-421 targeting E-cadherin in breast cancer [36]. Besides, Kruer et al. have reported that IncRNA MEG3 abnormal expression may affected lung cancer cell growth via Rb pathway [37]. In this research, these results indicated that CSE upregulated the IncRNA MEG3 expression in HPMECs.

Precious evidences have confirmed that IncRNA was dysregulated in many diseases, including COPD [38,39]. As human pulmonary microvascular endothelial cell apoptosis is essential in the progression of COPD $[20,21]$, we then attempted to investigate the effects of IncRNA MEG3 on the apoptosis of HPMECs. Many studies have explored the pathogenesis of COPD in vitro by studying the apoptosis of HPMECs $[20,21,23]$. Consistent with the previous findings [23], the data of the current study indicated that $1 \%$ CSE significantly induced apoptosis in HPMECs. In addition, the functional assay demonstrated that the upregulation of IncRNA MEG3 resulted in more apoptotic HPMECs, and higher caspase-3 activity in HPMECs. While IncRNA MEG3-shRNA led to fewer apoptotic HPMECs, and lower caspase-3 activity in HPMECs. These results demonstrated 
that InCRNA MEG3 played a vital role in COPD through regulating HPMEC apoptosis.

Furthermore, we then verified whether CSE promoted apoptosis in HPMECs by upregulating IncRNA MEG3, and rescue experiments were performed. The results revealed that the increased IncRNA MEG3 level, enhanced cell apoptosis, upregulated caspase- 3 activity, reduced $\mathrm{Bcl}-2$ level and enhanced Bax expression in HPMECs were significantly eliminated by InCRNA MEG3-shRNA.

All in all, these results evidenced that IncRNA MEG3 was participated in the evolution of COPD and might be strongly associated with cell apoptosis in HPMECs. Moreover, this study confirmed that IncRNA MEG3 might be an underlying target

\section{References:}

1. Fan W, Lee $\mathrm{H}$, Lee A et al: Association of lung function and chronic obstructive pulmonary disease with American Heart Association's Life's Simple 7 cardiovascular health metrics. Respir Med, 2017; 131: 85-93

2. Welte T: Chronic obstructive pulmonary disease - a growing cause of death and disability worldwide. Dtsch Arztebl Int, 2014; 111: 825-26

3. Lee JH, Cho MH, Hersh CP et al: Genetic susceptibility for chronic bronchitis in chronic obstructive pulmonary disease. Respir Res, 2014; 15: 113

4. Salvi S: Tobacco smoking and environmental risk factors for chronic obstructive pulmonary disease. Clin Chest Med, 2014; 35: 17-27

5. Liang Y, Chang C, Zhu H et al: Correlation between decrease of CRP and resolution of airway inflammatory response, improvement of health status, and clinical outcomes during severe acute exacerbation of chronic obstructive pulmonary disease. Intern Emerg Med, 2015; 10: 685-91

6. Hyman JJ, Reid BC: Cigarette smoking, periodontal disease: And chronic obstructive pulmonary disease. J Periodontol, 2004; 75: 9-15

7. Zeng $\mathrm{H}$, Kong $\mathrm{X}$, Peng $\mathrm{H}$ et al: Apoptosis and $\mathrm{Bcl}-2$ family proteins, taken to chronic obstructive pulmonary disease. Eur Rev Med Pharmacol Sci, 2012; 16: 711-27

8. He B, Chen Q, Zhou D et al: Role of reciprocal interaction between autophagy and endoplasmic reticulum stress in apoptosis of human bronchial epithelial cells induced by cigarette smoke extract. IUBMB Life, 2019; 71: 66-80

9. Ge J, Geng $\mathrm{S}$, Jiang $\mathrm{H}$ : Long noncoding RNAs antisense noncoding RNA in the INK4 locus (ANRIL) correlates with lower acute exacerbation risk, decreased inflammatory cytokines, and mild GOLD stage in patients with chronic obstructive pulmonary disease. J Clin Lab Anal, 2019; 33 e22678

10. Wu DM, Han XR, Wen X et al: Long non-coding RNA LINC01260 inhibits the proliferation, migration and invasion of spinal cord glioma cells by targeting CARD11 via the NF-kappaB signaling pathway. Cell Physiol Biochem, 2018; 48: 1563-78

11. Li P, Zhou B, Lv Y, Qian Q: LncRNA HEIH regulates cell proliferation and apoptosis through miR-4458/SOCS1 axis in triple-negative breast cancer. Hum Cell, 2019; 32(4): 522-28

12. Wu X, Yan T, Wang $Z$ et al: LncRNA ZEB2-AS1 promotes bladder cance cell proliferation and inhibits apoptosis by regulating miR-27b. Biomed Pharmacother, 2017; 96: 299-304

13. Ghafouri-Fard S, Taheri M: Maternally expressed gene 3 (MEG3): A tumor suppressor long non coding RNA. Biomed Pharmacother, 2019; 118: 109129

14. Chen J, Peng X, Dai Y: The long non-coding RNA (IncRNA) AGAP2-AS1 is upregulated in ovarian carcinoma and negatively regulates IncRNA MEG3. Med Sci Monit, 2019; 25: 4699-704

15. Xu J, Xu Y: The IncRNA MEG3 downregulation leads to osteoarthritis pro gression via miR-16/SMAD7 axis. Cell Biosci, 2017; 7: 16

16. Zhan R, Xu K, Pan J et al: Long noncoding RNA MEG3 mediated angiogenesis after cerebral infarction through regulating p53/NOX4 axis. Biochem Biophys Res Commun, 2017; 490: 700-6 for COPD therapy. However, we only investigated the function of InCRNA MEG3 in CSE-induced HPMECs in vitro model, more valid animal models need to be designed in future investigation.

\section{Conclusions}

LnCRNA MEG3 is participated in the evolution of COPD through regulating cell apoptosis of HPMECs. LncRNA MEG3 may be an underlying target for COPD therapy.

\section{Conflict of interests}

None.

17. Qiu GZ, Tian W, Fu HT et al: Long noncoding RNA-MEG3 is involved in diabetes mellitus-related microvascular dysfunction. Biochem Biophys Res Commun, 2016; 471: 135-41

18. Zhang S, Guo W: Long non coding RNA MEG3 suppresses the growth of glioma cells by regulating the miR $965 \mathrm{p} /$ MTSS1 signaling pathway. Mol Med Rep, 2019; 20: 4215-25

19. Zhang J, Gao Y: Long non-coding RNA MEG3 inhibits cervical cancer cell growth by promoting degradation of P-STAT3 protein via ubiquitination. Cancer Cell Int, 2019; 19: 175

20. Long YJ, Liu XP, Chen SS et al: miR-34a is involved in CSE-induced apoptosis of human pulmonary microvascular endothelial cells by targeting Notch-1 receptor protein. Respir Res, 2018; 19: 21

21. Chen Y, Wang H, Luo G, Dai X: SIRT4 inhibits cigarette smoke extracts-induced mononuclear cell adhesion to human pulmonary microvascular endothelial cells via regulating NF- $\kappa B$ activity. Toxicol Lett, 2014; 226: 320-27

22. Richter A, O'Donnell RA, Powell RM et al: Autocrine ligands for the epidermal growth factor receptor mediate interleukin-8 release from bronchia epithelial cells in response to cigarette smoke. Am J Respir Cell Mol Biol, 2002; 27: 85-90

23. Sun $\mathrm{Y}, \mathrm{An} \mathrm{N}$, Li J et al: MiRNA-206 regulates human pulmonary microvas cular endothelial cell apoptosisvia targeting in chronic obstructive pulmonary disease. J Cell Biochem, 2019; 120: 6223-36

24. Dima E, Kyriakoudi A, Kaponi $M$ et al: The lung microbiome dynamics between stability and exacerbation in chronic obstructive pulmonary disease (COPD): Current perspectives. Respir Med, 2019; 157: 1-6.

25. Noonan MC, Wingham J, Dalal HM, Taylor RS: Involving caregivers in selfmanagement interventions for patients with heart failure and chronic obstructive pulmonary disease. A systematic review and meta-analysis. J Adv Nurs, 2019; 75(12): 3331-45

26. Graham KG, Nasir A: Chronic cavitary pulmonary aspergillosis: A case report and review of the literature. Am J Case Rep, 2019; 20: 1220-24

27. Hutchinson D, Muller J, McCarthy JE et al: Cadmium nanoparticles citrullinate cytokeratins within lung epithelial cells: Cadmium as a potential cause of citrullination in chronic obstructive pulmonary disease. Int J Chron Obstruct Pulmon Dis, 2018; 13: 441-49

28. Liu SK, Chen P, Chen JB: [Apoptosis of alveolar epithelial cells and pulmonary vascular endothelial cells in chronic obstructive pulmonary disease]. Zhonghua Jie He He Hu Xi Za Zhi, 2008; 31: 581-85

29. Zhuo SM, Li SC, Lin YQ et al: The effects of anti-Fas ribozyme on T lymphocyte apoptosis in mice model with chronic obstructive pulmonary disease. Iran J Basic Med Sci, 2017; 20: 1102-8

30. Loeckx M, Rabinovich RA, Demeyer $\mathrm{H}$ et al: Smartphone-based physical activity telecoaching in chronic obstructive pulmonary disease: Mixedmethods study on patient experiences and lessons for implementation. JMIR Mhealth Uhealth, 2018; 6: e200 
31. Deng W, Yang W, Zeng J et al: Mycobacterium tuberculosis PPE32 promotes cytokines production and host cell apoptosis through caspase cascade accompanying with enhanced ER stress response. Oncotarget, 2016; 7: 67347-59

32. Wang LK, Chen XF, He DD et al: Dissection of functional IncRNAs in Alzheimer's disease by construction and analysis of IncRNA-mRNA networks based on competitive endogenous RNAs. Biochem Biophys Res Commun, 2017; 485: 569-76

33. Yu D, Tang C, Liu P et al: Targeting IncRNAs for cardiovascular therapeutics in coronary artery disease. Curr Pharm Des, 2018 [Epub ahead of print]

34. Herrera-Solorio AM, Armas-Lopez L, Arrieta $\mathrm{O}$ et al: Histone code and long non-coding RNAs (IncRNAs) aberrations in lung cancer: Implications in the therapy response. Clin Epigenetics, 2017; 9: 98
35. Wang J, Xu W, He Y et al: LncRNA MEG3 impacts proliferation, invasion, and migration of ovarian cancer cells through regulating PTEN. Inflamm Res, 2018; 67: 927-36

36. Zhang W, Shi S, Jiang J et al: LncRNA MEG3 inhibits cell epithelial-mesenchymal transition by sponging miR-421 targeting E-cadherin in breast cancer. Biomed Pharmacother, 2017; 91: 312-19

37. Wang M, Li C, Zhang Y et al: LncRNA MEG3-derived miR-361-5p regulate vascular smooth muscle cells proliferation and apoptosis by targeting ABCA1. Am J Transl Res, 2019; 11: 3600-9

38. Gu C, Li Y, Liu J et al: LncRNAmediated SIRT1/FoxO3a and SIRT1/p53 signaling pathways regulate type II alveolar epithelial cell senescence in patients with chronic obstructive pulmonary disease. Mol Med Rep, 2017; 15: 3129-34

39. Wang Y, Kong D: Knockdown of IncRNA MEG3 inhibits viability, migration, and invasion and promotes apoptosis by sponging miR-127 in osteosarcoma cell. J Cell Biochem, 2018; 119: 669-79 